\title{
Selected results from the ARGO-YBJ experiment
}

\author{
P. Camarri ${ }^{* \dagger}$ \\ University of Roma "Tor Vergata" and INFN Roma Tor Vergata \\ E-mail: paolo.camarridroma2.infn.it
}

The ARGO-YBJ experiment is an unconventional air-shower array designed for studying astronomical gamma-ray sources at energy greater than few hundred $\mathrm{GeV}$ and cosmic-ray physics at energy greater than about $1 \mathrm{TeV}$. The detector fulfils the requirements to achieve such goals thanks to its high-altitude location, 4300 meters a.s.l. on the Tibet plateau, and to its structure: a full-coverage layer of Resistive Plate Chambers (RPCs) covering a surface of $78 \times 74 \mathrm{~m}^{2}$, surrounded by a guard ring of RPCs enclosing a total surface of about $11000 \mathrm{~m}^{2}$. ARGO-YBJ has been running with the complete layout since November 2007, collecting about $4 \times 10^{11}$ events. The main results obtained by ARGO-YBJ, namely the cosmic-ray anisotropy, the monitoring of gamma-ray sources and the limit on the antiproton-proton ratio in the primary cosmic radiation, will be discussed.

36th International Conference on High Energy Physics,

July 4-11, 2012

Melbourne, Australia

\footnotetext{
* Speaker.

† on behalf of the ARGO-YBJ Collaboration.
} 


\section{Introduction}

The ARGO-YBJ experiment [ $[$ ] is a collaboration between Chinese and Italian research groups. It is located in Tibet, China, at an altitude of $4300 \mathrm{~m}$ a.s.l. in order to reconstruct air showers generated by cosmic rays with energy down to a few hundred GeV. ARGO-YBJ was designed to investigate a large number of topics in astrophysics and cosmic-ray physics: $\gamma$-ray astronomy (search for point-like sources above few hundreds of $\mathrm{GeV}$ ), search for VHE tails of $\gamma$-ray bursts above $\sim 1$ $\mathrm{GeV}$, cosmic-ray physics, Sun and heliosphere physics. The ARGO-YBJ detector is based on a full-coverage single layer of Resistive Plate Chambers (RPCs) covering a surface of $74 \times 78 \mathrm{~m}^{2}$. A group of 12 neighboring RPCs is called a "cluster", and the full-coverage part of the detector includes 130 clusters. Around this, 23 additional clusters are placed in order to obtain a better reconstruction of the shower front, which extends the experiment area to $110 \times 100 \mathrm{~m}^{2}$. The spacetime unit of the ARGO-YBJ detector is a "pad", namely a group of 8 neighboring RPC read-out strips. The single-hit time resolution is about $1.8 \mathrm{~ns}[[]$. Air showers are triggered by requiring a number of hits greater than 20 within a $150 \mathrm{~ns}$ time window, giving an average trigger rate of about $3.5 \mathrm{kHz}$ with a dead time of $4 \%$ and an average duty cycle greater than $86 \%$. ARGO-YBJ has been running almost uninterruptedly with its complete layout since October 2007 with a duty cycle of $90 \%$ and an average trigger rate of $3.6 \mathrm{kHz}$. A selection of the main physics results obtained so far is presented here.

\section{Results in $\gamma$-ray astronomy}

The analysis of the ARGO-YBJ data in $\gamma$-ray astronomy in the first three years of running was mainly focused on the study of known sources, on the study of AGN flares, and on the limits on the fluence from $\gamma$-ray bursts in the "Very High Energy" range (down to a few hundred $\mathrm{GeV}$ ). The background was evaluated by using two independent, widely established procedures: the "time-swapping" method and the "equi-zenith" method, which give equivalent results. The $\gamma$ ray emission from the Crab Nebula was detected with a significance of about 17 standard deviations in 4 years. Figure $\mathbb{⿴}$ shows the experimental points measured by ARGO-YBJ on the Crab Nebula energy spectrum, in perfect agreement with the known experimental results obtained by the HESS, MAGIC, Whipple and Veritas experiments.

A few remarkable flares from the AGN MRK421 were observed in the X-ray range by the ASM X-ray telescope in 2006, 2008 and 2010, and ARGO could observe TeV gamma emission at the same time for such flares, as shown in figure $\square$ for the two most recent ones.

Concerning the two flares observed on June 4-6 and June 11-13, 2008 [3]], ARGO-YBJ investigated the gamma emission from the same source on those days [䧃]. The peak significance for the flare of June 11-13, 2008 was 4.2 standard deviations. The ARGO-YBJ data fully satisfy the relation between the spectral index and the flux resulting from the Whipple measurements of a similar flare of MRK421 [5], suggesting that this relation is an intrinsic property of the source. One more remarkable flare from MRK421 occurred on February 2010. ARGO-YBJ observed it between February 16th and February 18th at a 6 s.d. significance level. The measured flux exceeded $3 \mathrm{Crab}$ units for the duration of the observation. The importance of this measurement by 


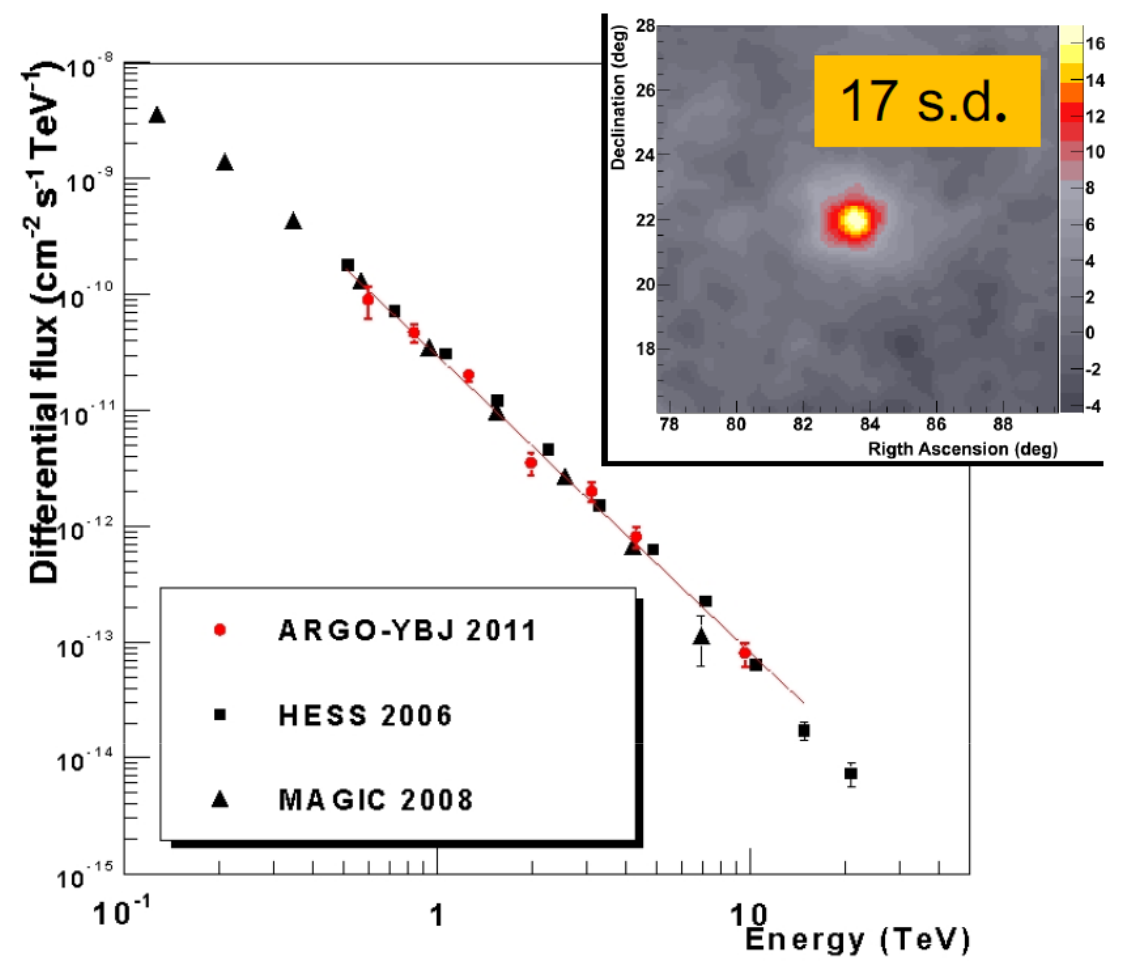

Figure 1: Comparison of the Crab $\gamma$-ray spectra measured by ARGO-YBJ (circles and power-law fit), HESS (squares) and MAGIC (triangles). The significance map of the Crab sky region measured by ARGO-YBJ between 2007 and 2011 is shown in the top-right corner.

ARGO-YBJ lies in the fact that for the first time a ground-based experiment could detect a flare with a 5 s.d. significance level on a daily basis.

An X-ray flare from the AGN MRK501 was detected by the RXTE/ASM telescope in October 2011 after a long "quiet" period (the previous intense X-ray flare dated back to 1997). This flare was associated to a strong emission in the TeV energy range detected by ARGO-YBJ [G]. During the flaring period, the $\mathrm{TeV}$ emission was observed in 36 days with a significance greater than 6 s.d.

At present ARGO-YBJ keeps performing a thorough full-sky search for more $\gamma$-ray sources and flaring phenomena.

\section{Results in cosmic-ray physics}

The studies in cosmic-ray physics with ARGO-YBJ were focused so far on the following items: search for anisotropy at $\mathrm{TeV}$ energies, study of the moon shadow and the corresponding limit on the $\bar{p}-p$ flux ratio, proton-air interaction cross section. Concerning the medium-scale anisotropy, the ARGO-YBJ significance map of the whole visible sky with cosmic rays is shown in Fig. B] [D]. 




Figure 2: Comparison of the cumulative counting rates for the $\gamma$-ray emission from the Crab nebula as measured by ARGO-YBJ (red), RXTE/AMS (black) and SWIFT (blue). The flaring periods are marked with circles. The vertical coordinates have been rescaled suitably in order to obtain a direct visual comparison of the three experimental results.

For this study, the data collected between 2007 and 2011 were used. A number of hit pads greater than 25 and a zenith angle $\theta<40^{\circ}$ were the requests for this analysis (corresponding to a median energy of about $1 \mathrm{TeV}$ ), and the map was obtained by using a smoothing window radius defined by the detector Point Spread Function (PSF). Four major excess regions are clearly visible. The regions 1 and 2 correspond respectively to the two excess regions of the all-sky significance map obtained by the MILAGRO experiment [8]. Therefore, ARGO-YBJ confirms the result obtained by MILAGRO for the medium-scale anisotropy. This effect is definitely surprising, since at the TeV energy scale the flux of galactic cosmic rays is expected to be highly isotropic due to the scrambling in the galactic magnetic field over a very long time. A possible explanation for this puzzling experimental evidence will be the subject of future studies.

An important subject of study in cosmic-ray physics is the shadowing effect exerted by the Moon on primary cosmic rays. Ground-based experiments can measure three crucial quantities related to this effect: the size, the position and the westward displacement of the deficit. From these measurements, information on the angular resolution, the pointing accuracy and the energy calibration of the detector respectively can be extracted. The angular resolution as a function of 


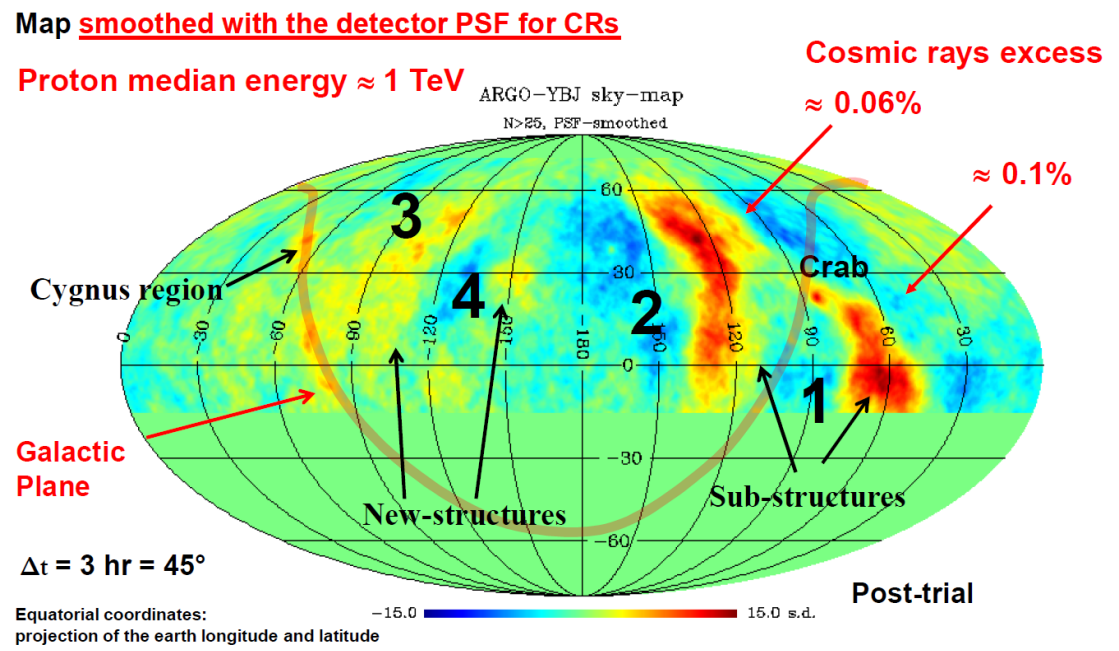

Figure 3: The ARGO-YBJ all-sky map, in equatorial coordinates. In this analysis (which uses the events with a hit multiplicity greater than 25) a smoothing was performed by using the detector Point Spread Function (PSF) for cosmic rays. The sky projection of the galactic plane is represented by the curved continuous line across the map. Four excess regions (peak significance greater than 5 s.d.) for the cosmicray flux are shown on the map.

the primary energy is a crucial feature of the experiment, and this is the reason which stresses the importance of this study. Concerning the last of these three issues, the basic procedure is based on the angular bending $\Delta \theta$ of a primary cosmic ray with energy $\mathrm{E}$ and atomic number $\mathrm{Z}$ in the geomagnetic field between the Moon and the Earth $\Delta \theta \simeq 1.57^{\circ} Z / E(\mathrm{TeV})$. The results coming from the data collected until the end of 2010 were described in detail in [Q]. A sky map of the cosmic-ray deficit due to the Moon measured by ARGO-YBJ is shown in Figure 4 .

A physical result which follows directly from the Moon-shadow analysis is the upper limit on the flux ratio of antiprotons and protons in the primary cosmic radiation at the $\mathrm{TeV}$ energy scale. ARGO-YBJ set an upper limit to $5 \%$ at a median energy of $1.4 \mathrm{TeV}$ and $6 \%$ at $5 \mathrm{TeV}$ with a confidence level of $90 \%[\mathbb{U}]$. These are the lowest upper limits available at the TeV scale.

\section{Conclusions}

The ARGO-YBJ experiment has been taking data almost uninterruptedly with its complete layout since November, 2007. It obtained important results in galactic and extragalactic $\gamma$-ray astronomy, and in cosmic-ray physics as well at the energy scale from $\sim 100 \mathrm{TeV}$ down to a few hundred GeV. More results are awaited in the near future.

\section{References}

[1] G. Aielli et al.; NIM A 562 (2006) 92-96.

[2] G. Aielli et al.; NIM A 608 (2009) 246-250. 




Figure 4: ARGO-YBJ sky map of the cosmic-ray flux deficit due to the Moon. This result was obtained with data collected between 2007 and 2011. The requirements for the events in this analysis were a hit multiplicity greater than 100 and a zenith angle less than $50^{\circ}$. The "Moon Shadow" was observed with a significance greater than 70 s.d. in 4 years.

[3] I. Donnarumma et al.; ApJ 691 (2009) L13-L19.

[4] G. Aielli et al. (the ARGO-YBJ collaboration); ApJ Letters 714 (2010) L208-L212.

[5] F. Krennrich et al. (the WHIPPLE collaboration); ApJ 575 (2002) L9-L13.

[6] B. Bartoli et al. (the ARGO-YBJ collaboration); ApJ 758 (2012) 2.

[7] R. Iuppa; Ph.D. thesis (University of Roma "Tor Vergata", 2012). G. Di Sciascio on behalf of the ARGO-YBJ collaboration; arXiv: 1010.4401.

[8] A. A. Abdo et al. (the MILAGRO collaboration); Phys. Rev. Lett. 101 (2008) 221101.

[9] B. Bartoli et al. (the ARGO-YBJ collaboration); Phys. Rev. D 84 (2011) 022003.

[10] B. Bartoli et al. (the ARGO-YBJ collaboration); Phys. Rev. D 85 (2011) 022002. 